\title{
Endofungal bacterium controls its host by an hrp type III secretion system
}

\author{
Gerald Lackner ${ }^{1}$, Nadine Moebius ${ }^{1}$ and Christian Hertweck ${ }^{1,2}$ \\ ${ }^{1}$ Leibniz Institute for Natural Product Research and Infection Biology (HKI), Department of Biomolecular \\ Chemistry, Beutenbergstr. 11a, Jena, Germany and ${ }^{2}$ Friedrich Schiller University, Jena, Germany
}

\begin{abstract}
Burkholderia rhizoxinica and Rhizopus microsporus form a unique symbiosis in which intracellular bacteria produce the virulence factor of the phytopathogenic fungus. Notably, the host strictly requires endobacteria to sporulate. In this study, we show that the endofungal bacteria possess a type III secretion system (T3SS), which has a crucial role in the maintenance of the alliance. Mutants defective in type III secretion show reduced intracellular survival and fail to elicit sporulation of the host. Furthermore, genes coding for T3SS components are upregulated during cocultivation of the bacterial symbiont with their host. This is the first report on a T3SS involved in bacterial-fungal symbiosis. Phylogenetic analysis revealed that the T3SS represents a prototype of a clade of yet uncharacterized T3SSs within the hrp superfamily of T3SSs from plant pathogenic microorganisms. In a control experiment, we demonstrate that under laboratory conditions, rhizoxin production was not required for establishment of the symbiotic interaction.

The ISME Journal (2011) 5, 252-261; doi:10.1038/ismej.2010.126; published online 19 August 2010

Subject Category: microbe-microbe and microbe-host interactions

Keywords: Burkholderia; Endofungal bacteria; Microbial interactions; Rhizopus; Symbiosis; Type III secretion
\end{abstract}

\section{Introduction}

Many bacterial strains live in close associations with eukaryotic hosts, ranging from invasive parasites to obligate mutualists having vital roles for their hosts (Moran, 2006). In mutualistic associations, specialized mechanisms have evolved that guarantee the persistence of the symbiosis. In particular, the transmission of the endosymbionts during host reproduction is a critical step for their survival. Impressive studies document the manifold strategies to ensure a permanent association of bacteria with partners of the animal and plant kingdoms (Dale and Moran, 2006; Moran, 2006; Bright and Bulgheresi, 2010). In stark contrast, nothing is known about the molecular basis of the transmission of endofungal bacteria, that is, bacteria that reside within the fungal cytosol (Kobayashi and Crouch, 2009; Tarkka et al., 2009). Recently, we have discovered an unprecedented toxinogenic alliance of the plant-pathogenic fungus Rhizopus microsporus and bacteria of the genus Burkholderia (Partida-Martinez and Hertweck, 2005). In this

Correspondence: C Hertweck, Friedrich Schiller University, Department of Biomolecular Chemistry, Leibniz Institute for Natural Product Research and Infection Biology (HKI), Beutenbergstr. 11a, Jena 07745, Germany.

E-mail: christian.hertweck@hki-jena.de

Received 3 May 2010; revised 14 June 2010; accepted 16 June 2010; published online 19 August 2010 highly specialized association, endobacteria supply their fungal host with the highly antimitotic compound rhizoxin, which serves as a virulence factor in rice seedling blight (Scherlach et al., 2006; Partida-Martinez and Hertweck, 2007; PartidaMartinez et al., 2007a, b; Lackner et al., 2009b). Interestingly, genetic studies indicated that the fungal host has gained insensitivity towards the antimitotic agent produced by the bacteria, thus implying a pathogen-mutualism shift during evolution (Schmitt et al., 2008). In fact, endosymbiontfree $R$. microsporus can be reinfected with isolated endobacteria under laboratory conditions. Furthermore, phylogenetic studies of a number of rhizoxinpositive isolates from all the five continents, delivered evidence for host switching of symbionts between fungal lineages (Lackner et al., 2009a), indicating that horizontal transmission has taken place during evolution of the symbiosis, and might still have a role in the natural environment. Yet, in each reproduction cycle of the fungus, $B$. rhizoxinica is passed on vertically to the next generation, which is a hallmark of close mutualistic relationships (Moran, 2006; Bright and Bulgheresi, 2010). Quite unexpectedly, we observed that the host is not capable of vegetative reproduction in the absence of endosymbionts. However, after reinfection of the fungal host, we observed endobacteria migrate into sporangia, enter vegetative spores of Rhizopus and survive therein until germination (Partida-Martinez 
et al., 2007b). This unique symbiont-dependent sporulation is an elegant way to prevent formation of symbiont-free spores, thus securing the persistence of the symbiosis. Our finding implies that the bacterial endosymbiont produces factors that are essential for fungal development and sporulation, and raises various questions regarding the molecular basis of this interaction. Bacteria living in symbiosis with eukaryotes, irrespective of whether they are parasites or mutualists, require specialized tools to control their host organisms. Prominent examples of such tools are type III secretion systems (T3SSs). These specialized protein export machineries form huge complexes spanning the inner and outer membrane of Gram-negative bacteria (Hueck, 1998; Ghosh, 2004; Cornelis, 2006). They are present both in human pathogens like Salmonella (Kubori et al., 1998) or Yersinia (Cornelis, 2002), and plant pathogens like Ralstonia solanacearum (Van Gijsegem et al., 1995; Genin and Boucher, 2004; Büttner and He, 2009). Typically, factors exported by T3SSs are effectors influencing the host organism during infection. Considering this knowledge, we were intrigued by the question whether a T3SS might be involved in maintenance or establishment of the Burkholderia-Rhizopus alliance.

In this study, we report that an ancient T3SS encoded in the bacterial genome has a key role in controlling this unique bacterial-fungal symbiosis.

\section{Results}

Fungal endosymbionts harbor genes coding for a T3SS To identify genes coding for protein secretion systems, we sequenced the genome of $B$. rhizoxinica using a whole genome shotgun approach. Within the obtained draft genome sequence we encountered a gene cluster encoding a T3SS on one of the sequence contigs. Further inspection confirmed that only one T3SS is encoded within the genome. The entire gene cluster spans about $22000 \mathrm{bp}$ (Figure 1) and contains 23 open reading frames (ORFs). In terms of primary sequence conservation and gene order, the gene cluster is similar to the hypersensitive response protein (hrp) locus coding for a T3SSs that promotes virulence of the plant pathogen Ralstonia solanacearum (Van Gijsegem et al., 1995, 2002; Genin and Boucher, 2004). Furthermore, it is similar to various yet obscure T3SS gene clusters, for example, T3SS 1 from Burkholderia pseudomallei K96243 (Rainbow et al., 2002; Warawa and Woods, 2005). Following suggestions from Hueck, (1998), we annotated the core components of the type III secreton as secretion and cellular translocation (sct) (Table 1). The deduced gene product of sctC is a well-conserved protein among animal and plant pathogens that belongs to the family of outer membrane ring proteins (secretins). SctU, SctV, SctR, SctS and SctT are conserved inner membrane proteins with several transmembrane helices representing essential parts of the secreton core structure. SctN, an ATPase, is located at the cytosolic side of the secreton and delivers energy for the secretion process (Ghosh, 2004; Cornelis, 2006). SctQ, an additional cytosolic protein, is likely involved in formation of a multimeric ring on the base of the injectisome (C-ring). Further genes within the cluster exhibit variable degrees of sequence conservation with $R$. solanacearum genes, which are only partly understood (Van Gijsegem et al., 2002).

Notably, the short predicted proteins SctE and SctF have no homology with any $R$. solanacearum gene product. However, from the location and length of their corresponding genes within the cluster it is likely that they are functional homologs of HrpX and HrpY, which are both essential components in the T3SS. HrpY is the pilin protein, the principal component of the Hrp pilus, and HrpX has a crucial role in pilus formation (Van Gijsegem et al., 2002). Whereas $S_{c t} E_{B r h}$ from B. rhizoxinica shows low homology to $\mathrm{Sct}_{\mathrm{Bps}}$ from B. pseudomallei, the proposed pilus protein $\left(\mathrm{SctF}_{\mathrm{Brh}}\right)$ has no sequence similarity to any protein in publicly available databases. Nevertheless, the high proportion of $\alpha$-helix structures predicted by PSIPRED supports the assumption that SctF might be the functional homolog of HrpY. Two ORFs (ORF 3 and 4), which are absent in all related gene clusters appear to be inserted into the T3SS cluster during evolution.

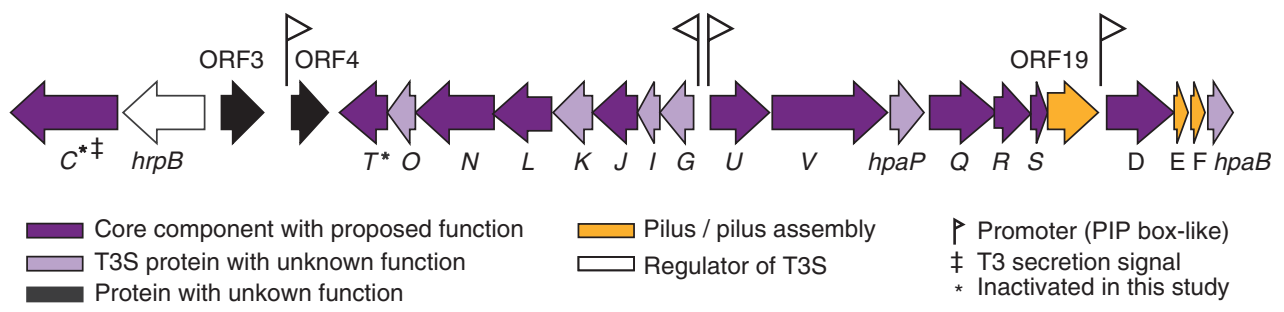

Figure 1 Schematic illustration of the type III secretion gene cluster of Burkholderia rhizoxinica. Arrows represent protein-coding regions. Genotype symbols represented by capital letters are abbreviations for the sct genes. Violet: Conserved genes encoding T3SS core components with homology to functionally well-characterized reference T3SSs. Grey: Genes involved in type III secretion without functionally characterized homologs. Yellow: Genes likely involved in pilus formation, with SctF being the putative pilin protein. White: Transcriptional regulator of T3S. T3 secretion signal was predicted with the EFFECTIVE T3 software. The nucleotide sequence of the T3SS gene cluster has been deposited in the EMBL database under the accession number FN687446. 
Table 1 Annotation of the B. rhizoxinica T3SS gene cluster

\begin{tabular}{|c|c|c|c|c|}
\hline ORF & Gene & Proposed function & R. solanacearum homolog & Percent identity (BLASTP) \\
\hline 1 & sctC & Outer membrane ring (secretin) & hrcC & 41 \\
\hline 2 & hrpB & Regulator & hrpB & 37 \\
\hline 3 & & Unknown & & - \\
\hline 4 & & Unknown & & - \\
\hline 5 & $\operatorname{sct} T$ & Inner membrane protein & hrcT & 43 \\
\hline 6 & sctO & Unknown & $h r p D$ & - \\
\hline 7 & $\operatorname{sct} N$ & ATPase & hrcN & 71 \\
\hline 8 & sctL & ATPase-associated regulator & hrpF & 31 \\
\hline 9 & $s c t K$ & Unknown & hrpH & - \\
\hline 10 & sctJ & Inner membrane ring (lipoprotein) & hrcJ & 58 \\
\hline 11 & sctI & Unknown & hrpJ & - \\
\hline 12 & $\operatorname{sct} G$ & Unknown & hrpK & - \\
\hline 13 & $\operatorname{sct} U$ & Inner membrane protein & hrcU & 47 \\
\hline 14 & $\operatorname{sct} V$ & Inner membrane protein & $h r c V$ & 59 \\
\hline 15 & hpaP & Unknown & hpaP & 35 \\
\hline 16 & $\operatorname{sct} Q$ & Putative cytosolic ring & hrcQ & 29 \\
\hline 17 & $\operatorname{sct} \widetilde{R}$ & Inner membrane protein & $h r c R$ & 63 \\
\hline 18 & $\operatorname{sct} S$ & Inner membrane protein & hrcS & 59 \\
\hline 19 & & Pilus assembly & $h r p V$ & - \\
\hline 20 & $\operatorname{sct} D$ & Inner membrane ring & hrpW & 26 \\
\hline 21 & sctE & Pilus assembly & hrpX & - \\
\hline 22 & $\operatorname{sctF}$ & Pilus & hrpY & - \\
\hline 23 & hpaB & Unknown & hраB & 56 \\
\hline
\end{tabular}

However, their function is unknown. Furthermore, we analyzed the gene cluster for promoter regions containing plant-inducible promoter boxes (Mukaihara et al., 2004). We identified plant-inducible promoter box-like motifs (TTCG-N ${ }_{12-18}$-TTCG) in promoter regions of sctD, sctG, sctU and ORF 4. This finding suggests that the corresponding operons might be under control of the HrpB transcriptional activator, and ORF 4 might code for a protein involved in T3S. In addition, we examined the $\mathrm{N}$-termini of all gene products for T3S signals (Arnold et al., 2009). As expected, the putative secretin SctC was found to harbor a secretion signature. However, ORF 4 was not predicted to be a T3 secreted effector by the prediction algorithm.

The B. rhizoxinica T3SS is a member of the hrp familiy To gain further insights into the evolutionary origin of the $B$. rhizoxinica T3SS gene cluster, we constructed a phylogenetic tree based on the conserved SctC component (Figure 2). According to the cladogram, the nearest relative of SctC was found in Burkholderia graminis C4D1M (accession ZP 02881763.1), a ubiquitous rhizosphere colonizer (Viallard et al., 1998). To our knowledge the function of this gene cluster is unknown. In a gene cluster comparison, we examined conservation of gene order and sequence homology among the $B$. rhizoxinica cluster, the T3SS 1 cluster from $B$. pseudomallei and the $R$. solanacearum hrp gene cluster by blasting all the protein sequences against each other. (Supplementary Figure S1). As expected, the core proteins are conserved among all clusters. However, some proteins are only conserved between the two Burkholderia clusters in terms of primary sequence homology. Among those is the putative pilus formation protein SctE. The only gene products that are conserved exclusively among $R$. solanacearum and $B$. pseudomallei are the putative pilus proteins $\mathrm{SctF}_{\mathrm{BPS}}$, $\mathrm{HrpY}$ and the pilusassociated HrpZ. This might be an indication that $\mathrm{SctF}_{\mathrm{BRH}}$ from $B$. rhizoxinica has undergone more extensive mutation due to adaptation to a different host species.

\section{T3SS mutants are unable to reestablish a stable symbiosis}

To address the question whether endosymbionts require the T3SS for interaction with their fungal host, we generated mutants defective in T3S. We modified methods for targeted gene inactivation previously applied to mutate $B$. thailandensis (Barrett et al., 2008) to match the requirements of the frail endosymbionts grown in pure culture. To avoid polar effects, we targeted genes sctC and sct $T$, both located at the end of putative transcription units. Although the introduction of plasmid DNA and homologous recombination was feasible, the effectiveness of the counter-selection step in the cultured symbionts was much lower than reported for B. thailandensis. However, after several weeks or months of selection we finally succeeded in the isolation of allelic replacement mutants for sctC and sctT (Supplementary Figure S2). Both the mutants $\left(\Delta s c t C:: \operatorname{Kan}^{\mathrm{r}}\right.$ and $\left.\Delta s c t T:: \operatorname{Kan}^{\mathrm{r}}\right)$ did not show any growth defects or morphological phenotypes compared with the wild type when grown under laboratory conditions. 

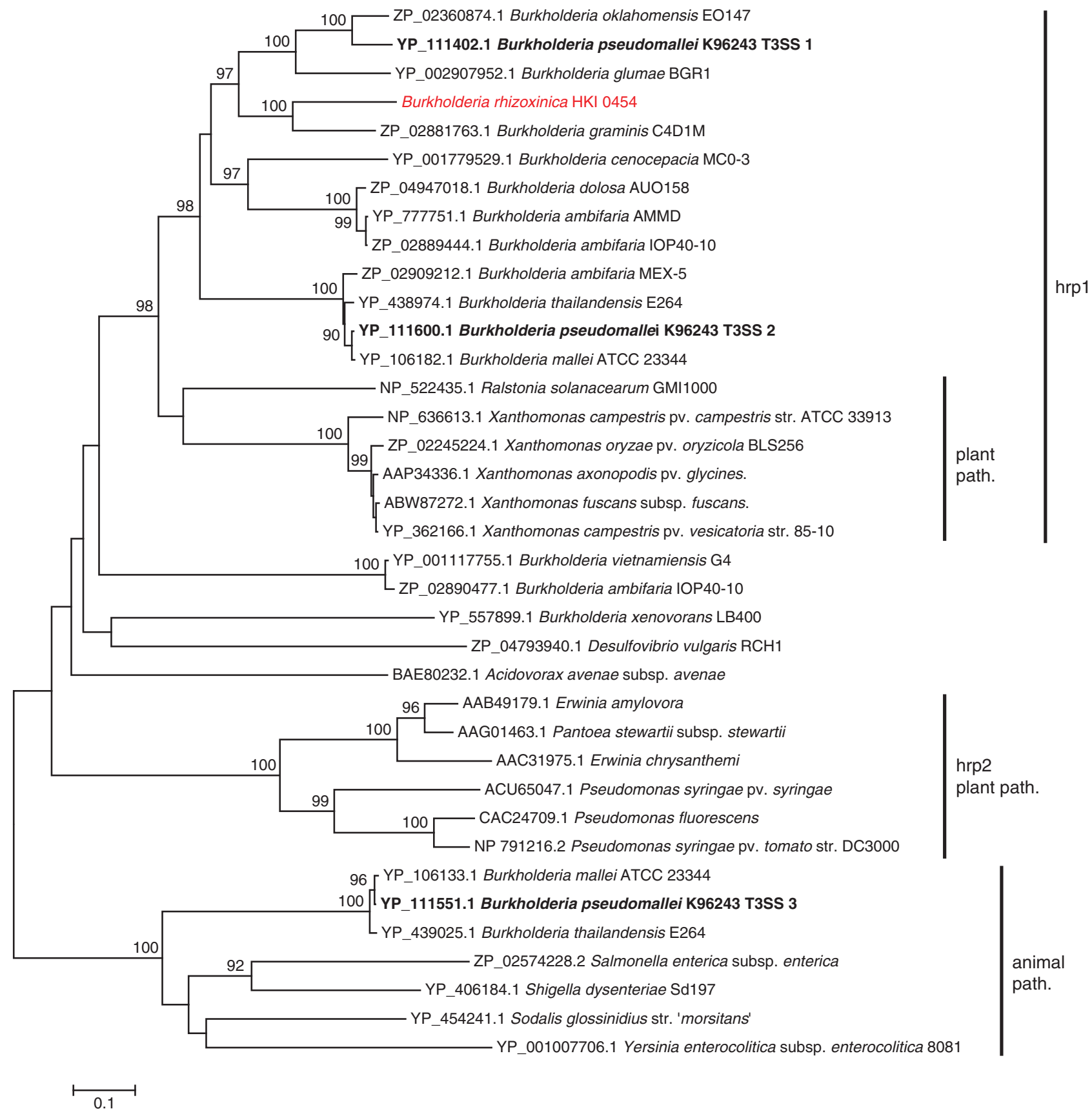

Figure 2 Neighbor-joining tree of SctC (outer membrane secretin) homologs in Burkholderia and selected Gram-negative plant and animal specific pathogens and symbionts. Accession numbers and source organisms are indicated on leaves. Significant bootstrap values are shown above branches. Components of $B$. pseudomallei type III secretion systems (T3SS clusters 1-3) are marked in bold letters. Hrp1: Family of T3SS known from plant pathogens including Ralstonia solanacearum and Xanthomonas campestris. Hrp2: Family of T3SSs found in plant pathogens like Pseudomonas syringae and Erwinia species. Animal path: T3SSs from human/animal symbionts and pathogens.

To examine the impact of genomic mutations on bacterial-fungal interaction we developed a new bioassay exploiting the capacity of endosymbionts to reinfect the host and restore fungal sporulation. Typically, during cocultivation of isolated wild-type endobacteria with cured fungi, bacteria reinfect their host, replicate within the fungal mycelia and finally recover the ability of the host to form sporangiospores. As this can be easily monitored by eye, host sporulation is an excellent indicator for the successful reconstitution of the symbiosis. We tested various cocultivation conditions for the assay both on agar plate and in liquid culture to obtain high rates of reinfection and sporulation using the $B$. rhizoxinica wild type strain.

Testing the T3SS mutants, sctC:: $\operatorname{Kan}^{\mathrm{r}}$ and $\Delta s c t T:: \mathrm{Kan}^{\mathrm{r}}$, we found that the T3SS mutants lack the ability to trigger sporulation of their host in an 

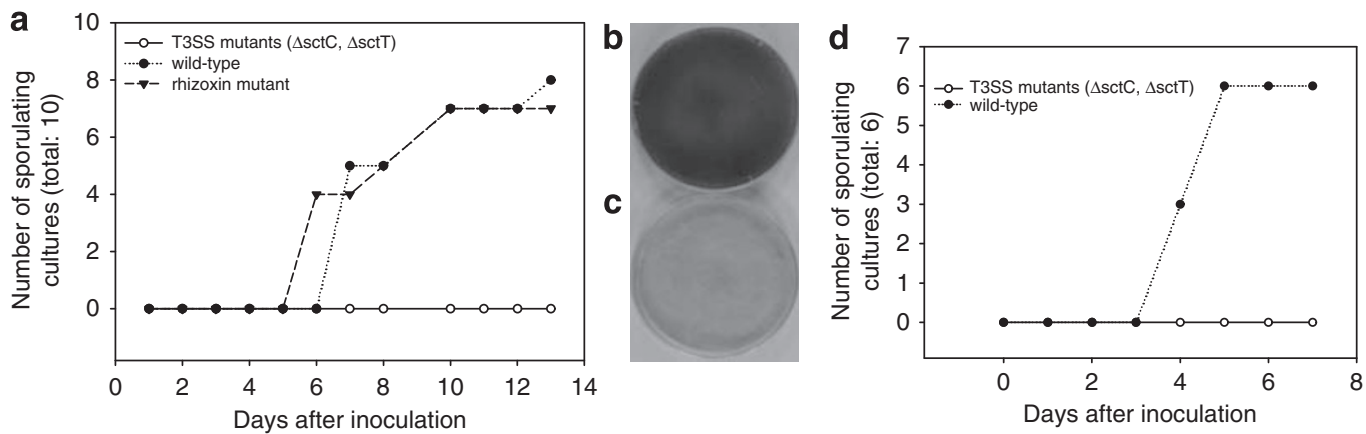

Figure 3 Reinfection/sporulation assay. Cured fungi were cocultivated with previously isolated B. rhizoxinica endosymbionts and sporulation of fungal cultures monitored by eye and light microscopy. Using wild type bacteria, after 5 days (agar plate) or 3 days (liquid media), fungal cultures start to sporulate, indicating successful reinfection and triggering of sporulation by endosymbionts. A rhizoxin biosynthesis deficient mutant shows identical behavior as the wild type. T3SS mutants $\left(\Delta s c t C:: \operatorname{Kan}^{\mathrm{r}}, \Delta s c t T:: \mathrm{Kan}^{\mathrm{r}}\right)$ failed to elicit host sporulation. (a): Reinfection/sporulation assay on agar plates. (b): Sporulating $R$. microsporus infected by wild type bacteria. (c): Non-sporulating $R$. microsporus infected with T3SS mutant. (d): Reinfection/sporulation assay in liquid media (48-well plates).

a
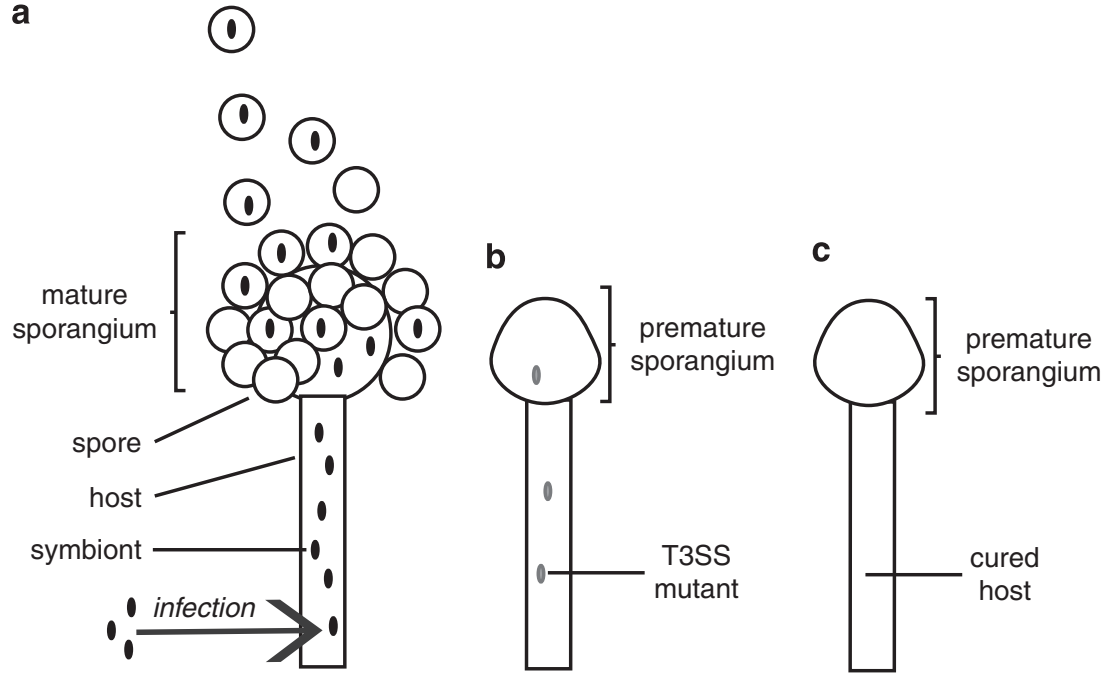

Figure 4 Schematic illustration of reinfection/sporulation assay. (a): wild type bacteria infect cured fungi, migrate into sporangia, trigger formation of sporangiospores and enter the spores. (b): T3SS mutants enter hyphae, but reach lower cell densities and fail to elicit sporulation. (c): cured fungi are incapable of forming mature sporangia and sporangiospores. Premature sporangia can be detected sporadically.

infection/sporulation assay (Figure 3). In contrast, both the wild-type strain and a mutant deficient in rhizoxin biosynthesis readily trigger sporulation both on agar plate and in liquid culture. In a control, where only MGY medium was added to the cured fungus, no spore formation was observed. Through visualization of bacteria with a live/dead stain we found that the majority of bacteria was alive for even 7 days after inoculation. Microscopic examination of fungal mycelium cocultivated with T3SS mutants revealed further important details. Typically, wildtype bacteria spread among the fungal mycelia quickly and reach considerable cell densities within the hyphae. After 3-7 days the host responds with the formation of mature sporangia carrying sporangiospores. In case of the T3SS mutants, however, no infection or only limited zones of infection were visible (Supplementary Video S1 and S2). As in cured fungal cultures, the host does not form spores, although premature sporangia can be detected sporadically (Figure 4). The finding that a rhizoxindeficient mutant acts like the wild type in a reinfection/sporulation experiment, finally proves that rhizoxin is neither necessary for the infection process nor for elicitation of sporulation.

\section{Bacterial T3SS genes are upregulated during} cocultivation with fungal host

The salient involvement of the type III secretion apparatus in symbiont-host interaction raised the question whether the expression of T3SS genes depends on the presence of the fungus (Figure 5). Using specific primers targeting sctC and sctT we monitored gene expression levels using quantitative real-time PCR in pure culture and during cocultivation of $B$. rhizoxinica with cured $R$. microsporus. Triplicate experiments unequivocally demonstrated 


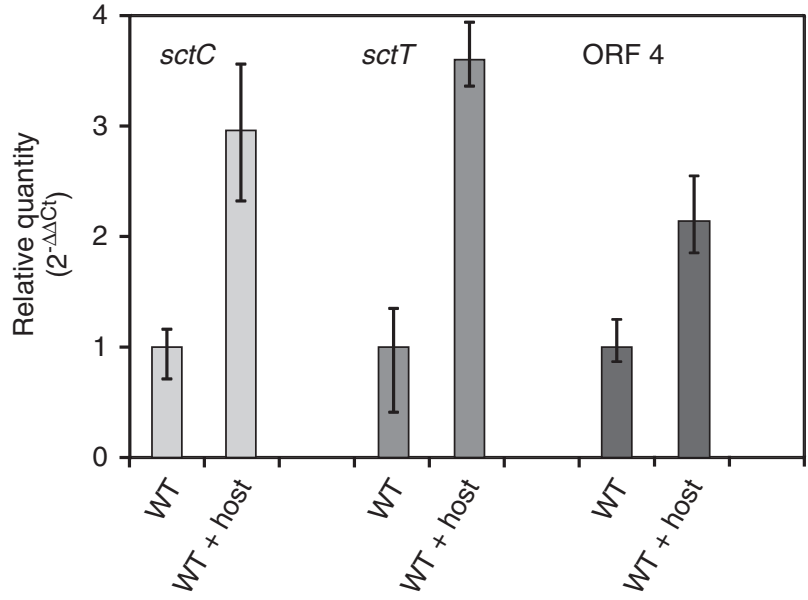

Figure 5 T3SS gene expression assay. Expression of T3S genes sctC (left) and sct $T$ (middle) and ORF 4 (right) in $B$. rhizoxinica monitored by quantitative real-time PCR both in pure culture and in cocultivation with cured host (R. microsporus). The $16 \mathrm{~S}$ gene was used as an internal standard for calculation of expression levels and normalization. Expression of both genes is significantly increased in the wild type (WT) during cocultivation.

that expression of both genes is significantly upregulated during cocultivation with a threefold increase for $s c t C$ and a 3.5-fold increase for sctT. These results clearly support the hypothesis that a T3SS is involved in bacterial-fungal interaction. Furthermore, we examined expression of the inserted genes ORF 3 and ORF 4. Although ORF 4, which is presumably under control of a putative plantinducible promoter box-like promoter showed a twofold upregulation during cocultivation, we could not find increased levels of mRNA for ORF 3 (data not shown). For this reason we hypothesize that the gene product of ORF 4 is a promising candidate for a novel T3 effector or helper protein.

\section{Discussion}

Burkholderia rhizoxinica is a vertically and horizontally transmitted intracellular endosymbiont of $R$. microsporus. In this unusual symbiosis, the bacterial endosymbionts produce an antimitotic toxin that serves its fungal host as a pathogenicity factor against rice plants. Although the host benefits from the biosynthetic capacities of its symbiont, it has lost its ability to reproduce autonomously and fully depends on the presence of its symbiont (Partida-Martinez et al., 2007b). As vegetative reproduction of the host has become totally dependent on the presence of the endosymbiont, persistence of the symbiosis is warranted. The factors and mechanisms triggering fungal sporulation have remained elusive to date. Various studies with nonfungal hosts have revealed that bacteria living in close association with higher organisms may use specialized tools like T3SSs to control their hosts.
Type III secreted effectors are required for processes like host cell invasion, inhibition of the host immune system (Grant et al., 2006) or extraction of nutrients from the host (Poueymiro and Genin, 2009). Notably, the action of type III secretion is not limited to parasitic or antagonistic associations. For instance rhizobia, nitrogen fixing mutualists of plants (Deakin and Broughton, 2009) or symbionts of tsetse fly and grain weevils, harbor T3SSs for interaction with their hosts (Dale et al., 2001, 2002). However, the role of T3SSs in bacterialfungal interactions has not yet been addressed (Preston, 2007; Kobayashi and Crouch, 2009). The only example known to date in which a T3SS is specific for a host not belonging to the kingdoms of animals or green plants is the T3SS of Pseudomonas fluorescens $\mathrm{KD}$ targeting the phytopathogenic chromista Pythium ultimum (Rezzonico et al., 2005). In this system, the bacterial biocontrol strain uses a T3SS to protect cucumber by reducing virulence of the oomycete parasite.

In this study we present the first gene cluster encoding a T3SS in the genome of an endofungal bacterium, B. rhizoxinica, and show that it is necessary for the establishment of a stable symbiosis. Exploiting the ability of wild-type endosymbionts to reinfect their host and trigger sporulation, we have developed a new bioassay that enabled us to test mutants for defects in symbiotic lifestyle. By applying a double-crossover mutagenesis strategy, we generated mutants deficient in type III secretion that fail to induce host sporulation after reintroduction into $R$. microsporus. Microscopic examination suggests that the intracellular survival rate is reduced compared with wild type bacteria. In addition, genes encoding core components of the T3SS, as well as a novel T3S gene (ORF 4) are upregulated during cocultivation with the host, supporting the idea that type III secretion is employed for bacterial-fungal interaction.

We assume the T3SS exports factors that promote infection, subdue defense mechanisms or assist in gaining nutrients from the host as it is known from plant pathogens (Grant et al., 2006; Poueymiro and Genin, 2009). Yet knowledge on mechanisms that allow fungi to actively prevent bacterial infection is scarce (Kobayashi and Crouch, 2009). Physical barriers, as well as the sequestration of nutrients or trace elements might protect the fungal mycelia from a number of uninvited guests. Furthermore, it was proposed that fungi posses a rudimentary innate immune system, including the capacity to produce chemical defense chemicals like antibiotics or reactive oxygen species (Kobayashi and Crouch, 2009). Our ongoing studies on type III effectors in the Burkholderia-Rhizopus symbiosis might shed more light on these processes that are still obscure.

In showing that a rhizoxin-deficient mutant behaves like the wild type in a reinfection/sporulation assay, we prove that the polyketide is not 
required for maintenance of the symbiosis under laboratory conditions. Considering that rhizoxin represents an essential benefit for the host organism in the natural habitat, we have generated a 'blind passenger' by re-introducing a non-producing Burkholderia mutant into the host. A 'blind passenger' is formed in evolution when a mutualist turns into a more parasitic symbiont by losing some of its beneficial properties without being eliminated from the host. This means that external selecting pressures acting on the symbiosis rather than intrinsic control mechanisms of the host have prevented $B$. rhizoxinica from turning into a parasitic symbiont by losing its biosynthetic capability.

Considering that diffusible low-molecular-weight metabolites do not seem to mediate sporulation (Partida-Martinez et al., 2007b), our results are in agreement with a model where the sporulation trigger itself is secreted by the type III apparatus. We propose that the endosymbionts have acquired or mimic a host factor that is necessary to launch its vegetative reproduction program. This factor must be present in Rhizopus strains that are naturally endosymbiont-free and capable of autonomous sporulation. In contrast, this factor might have been lost or is permanently silenced in Rhizopus strains that strictly depend on their endosymbionts. Molecular mimicry of signal transduction proteins or transcription factors is an elegant strategy known from pathogens to elicit desired host responses (Zhou and Chai, 2008). Our results deliver a promising starting point for the identification of the sporulation triggers.

Notably, the T3SS presented in this study is the first secretion system shown to act on a fungal host. Surprisingly, phylogenetic analyses revealed that it belongs to an ancient family of T3SSs, which are known from plant pathogens like $R$. solanacearum (Genin and Boucher, 2004). It is remarkable that the mycota-specific T3SS is more related to the plantspecific Hrp apparatus than to the injectisomes known from human or animal pathogens. We suspect that this might be partly because of the fact that a Hrp pilus structure is more suited to overcome a cell wall, a feature that is shared by both mycota and plants. The finding that the $B$. rhizoxinica T3SS is related to the T3SS found in the genome of the rhizosphere colonizer $B$. graminis and the T3SS 1 of $B$. pseudomallei inspires new hypotheses about the functional role of these yet obscure gene clusters. Unlike the animal pathogen-like TTSS 3, the apparatus encoded by T3SS 1 is not necessary for elicitation of melioidosis, a disease caused by B. pseudomallei in humans (Kim et al., 2005; Warawa and Woods, 2005). It may, however, neither be a plant-specific secretion system as suggested before (Warawa and Woods, 2005). In contrast, considering that many Burkholderia strains share habitats with fungal species (Coenye and Vandamme, 2003), we predict that some of the uncharacterized T3SSs may be specific for a fungal host.
In conclusion, we have cloned and sequenced a gene cluster coding for a T3SS in the bacterial endosymbiont of the phytopathogenic fungus $R$. microsporus. Targeted gene knockouts of key genes (coding for SctC and SctT) resulted in a phenotype that is incapable of forming a stable symbiosis with the fungus. Intriguingly, the mutants lost their ability to stimulate sporulation in the host, revealing an unprecedented case where a T3SS is critical for controlling the physiology of a fungal host. Remarkably, the T3SS genes are clearly upregulated in a bacterial-fungal coculture. Finally, phylogenic analyses revealed that the $B$. rhizoxinica T3SS is distant from animal/human pathogens and represents the prototype of a clade of yet uncharacterized T3SSs within a superfamily of T3SSs from plant pathogenic microorganisms. Thus, the finding of the first T3SS in a bacterial-fungal mutualism closes an important gap in the large body of knowledge on type III secretion in symbioses.

\section{Materials and methods}

\section{Strains and culture conditions}

Rhizopus microsporus (ATCC62417) harboring endobacteria $B$. rhizoxinica (isolate B1, HKI 0454) was used in this study. Pure cultures of B. rhizoxinica were grown in MGY medium (M9 minimal medium supplemented with $1.25 \mathrm{gl}^{-1}$ yeast extract and $10 \mathrm{gl}^{-1}$ gycerol) at $30^{\circ} \mathrm{C}$. Isolation of endobacteria (Partida-Martinez and Hertweck, 2005), preparation of electrocompenent cells and curation of the fungus from endosymbionts (Partida-Martinez et al., 2007b) were conducted as described before. Wild type and cured strains of $R$. microsporus were grown on potato dextrose agar at $30^{\circ} \mathrm{C}$.

\section{Generation of T3SS mutants of B. rhizoxinica}

For the construction of a suicide-vector containing a counter-selectable marker, a mutated phenylalanyl tRNA synthetase gene pheS (accession number EU277853) was used. The PheS gene product is toxic in the presence of 4-L-chlorophenylalanine. Thus, cPhe can be used to select for double-crossover mutants that have eliminated the pheS gene (Gamper and Kast, 2005). To avoid recombination with the natural pheS gene, the coding sequence has been modified (Barrett et al., 2008). The artificial gene was synthesized (GenScript, Piscataway, NJ, USA) and ligated into the NspI site of pBlueskript II $(S K+)$ yielding vector pGL42a. For the construction of the $\Delta s c t C:: \operatorname{Kan}^{\mathrm{r}}$ mutant a region upstream of sctC (973 bp) was amplified using Pfu polymerase in 35 cycles $\left(95^{\circ} \mathrm{C}\right.$ for $15 \mathrm{~s}, 58^{\circ} \mathrm{C}$ for $30 \mathrm{~s}$ and $72^{\circ} \mathrm{C}$ for 2 min) with oligonucleotides P1_sctC (Table 2) and P2_sctC to obtain PCR product Sct_1. A region downstream of $s c t C$ (1104 bp) was amplified using oligonucleotides P3_sctC and P4_sctC to yield PCR product Sct_2. Equimolar amounts of PCR products 
Table 2 Oligonucleotides used in this study

Kan NheI

Kan_PacI

P1_sctC

P2_sctC

P3_sctC

P4_sctC

P1_sctT

P2_sctT

P3_sctT

P4 sctT

sctC_int_for

sctC_int_rev

sctT_int_for

sctT_int_rev

qRTsctC_fw

qRTsctC_rw

qRTsctT_fw

qRTsctT_rw

\author{
5'-GCTAGGCTAGCGGATGAATGTCAGCTACTGGGC-3' \\ 5'-GCTAGTTAATTAATCAGAAGAACTCGTCAAGAAGGC-3' \\ $5^{\prime}$-GCTAGGCGGCCGCCGCTGGCAGGAGGTTGT-3' \\ 5'-GATCGTTAATTAAGCTAGCCTAGCACGGCGATCGAAGGGTAT-3 ${ }^{\prime}$ \\ $5^{\prime}$-GCTAGGCTAGCTTAATTAACGATCTGTCACCGCGCATCATTGAATT- ${ }^{\prime}$ \\ $5^{\prime}$-GCTAGGGGCCCAATTCCGCTTAGCGAGACAA- ${ }^{\prime}$ \\ 5'-GCTACGGTACCTTGCAGCGAGGAACCAGTAT-3' \\ 5'-GCTACTTAATTAAGCTAGCGTAGCGATCTCCAATAGCCCGTTCA-3' \\ 5'-TGCTACGCTAGCTTAATTAAGTAGCTGTTCGTTGGCATCTTTGTC-3' \\ $5^{\prime}$-GCTACGAGCTCCCACCTGAAGACCGTCAGAA-3' \\ $5^{\prime}$-TGAACTGGATGCAGACAACC-3' \\ $5^{\prime}$-CCTGGCTAGACGCAAAGTCT- ${ }^{\prime}$ \\ $5^{\prime}$-AAAGTGCCGGAACGTACATC- $3^{\prime}$ \\ $5^{\prime}$-CGTATCCGTTTGATGCAAT- $3^{\prime}$ \\ $5^{\prime}$-CTTGGTCGTGGAGGAGGAC- $3^{\prime}$ \\ $5^{\prime}$-AACGCTTCGGTATTGATGG-3' \\ $5^{\prime}$-TCAGCACAGAAGCTGGAAGT- $3^{\prime}$ \\ 5'-CATGTAGTCCCCCAATCCTG-3'
}

Sct_1 (250 ng) and Sct_2 (283 ng) were used as templates for an overlapping PCR. Overall, 10 PCR cycles $\left(95^{\circ} \mathrm{C}\right.$ for $15 \mathrm{~s}, 52^{\circ} \mathrm{C}$ for $30 \mathrm{~s}$ and $72{ }^{\circ} \mathrm{C}$ for $2 \mathrm{~min}$ ) were carried out without primers to join template molecules. After 10 cycles, additional 25 cycles $\left(95^{\circ} \mathrm{C}\right.$ for $15 \mathrm{~s}, 58{ }^{\circ} \mathrm{C}$ for $30 \mathrm{~s}$ and $72^{\circ} \mathrm{C}$ for $3 \mathrm{~min}$ ) were run including oligonucleotides P1_sctC and P4_sctC to amplify the overlap product. The obtaine $\bar{d}$ PCR product stc_4 was cut using NotI and ApaI and ligated into pBluescript II $(\mathrm{SK}+)$ yielding pGL38. The kanamycin-resistance cassette from vector pK19 was amplified using primers Kan_NheI and Kan_PacI and ligated into the insert of pGL38 yielding pGL39. The obtained insert (3289 bp) was restricted with $\mathrm{KpnI} / \mathrm{SacI}$ and ligated into vector pGL42a yielding pGL45. For the construction of the $\Delta s c t T:: \mathrm{Kan}^{\mathrm{r}}$ mutant, oligonucleotides P1_sctT and P2_sctT, as well as P3_sctT and P4_sctT were used for PCR reactions to obtain PCR products sctT_1 (883 bp) and sctT_2 (958 bp), respectively. Both products were used as templates for an overlapping PCR as described before. The obtained overlap product was digested with $\mathrm{KpnI} / \mathrm{SacI}$ and directly ligated into pGL42a yielding pGL56. To obtain the knockout vector pGL57 the kanamycin cassette was ligated into the NheI/PacI restriction sites in pGL56. Vectors pGL45 and pGL57 were introduced into competent cells of $B$. rhizoxinica by electroporation. Transformants were selected on standard nutrient agar supplemented with $50 \mu \mathrm{g} \mathrm{ml}^{-1}$ kanamycin. Surviving colonies were grown in liquid MCGAVT medium (M9 minimal media containing $10 \mathrm{gl}^{-1}$ glycerol, amino acid mix without phenylalanin, vitamin solution, trace elements, $50 \mu \mathrm{g} \mathrm{ml}^{-1}$ kanamycin and $2 \mathrm{gl}^{-1}$ 4-D,L-chlorophenylalanine) for 3 days, spread on MCGAVT agar plates to obtain single colonies and checked for remaining wild type contamination by PCR using Taq polymerase running for 35 cycles $\left(95^{\circ} \mathrm{C}\right.$ for $15 \mathrm{~s}, 54{ }^{\circ} \mathrm{C}$ for $15 \mathrm{~s}$ and $68^{\circ} \mathrm{C}$ for $30 \mathrm{~s}$ ). For detection of $s c t C$ we amplified a $216 \mathrm{bp}$ fragment using primers sctC_int_for and
sctC_int_rev, for sct T a $254 \mathrm{bp}$ fragment using sctT_int_for and sctT_int_rev. The selection process was repeated until a mutant missing the wild type gene fragment could be identified. Finally, we confirmed genotypic integrity of mutants by PCR spanning both recombination sites (see Supplementary Material).

\section{Reinfection/sporulation assay for bacterial-fungal interaction}

Bacterial cells were grown in MGY media without antibiotics until they reached an $\mathrm{OD}^{600}=1.5$. For reinfection/sporulation assay on agar plate, a $100 \mu \mathrm{l}$ cell suspension was plated on one half of a nutrient agar plate $(60 \mathrm{~mm} \varnothing)$. The second half of the plate was inoculated with cured (endosymbiont-free) R. microsporus ATCC62417. Ten plates each of wild type and mutant strain were prepared in parallel. The plates were dried well and incubated at $30^{\circ} \mathrm{C}$. For an assay in liquid media, 48-well plates were used. Each well was filled with $800 \mu \mathrm{l}$ VK-medium (1\% corn starch, $0.5 \%$ glycerol, $1 \%$ yeast extract, $1 \%$ corn steep and $\left.1 \% \mathrm{CaCO}_{3}, \mathrm{pH}=6.5\right)$ and inoculated with about $0.1 \mathrm{~cm}^{3}$ of aerial mycelium of $R$. microsporus. Subsequently, $100 \mu$ of bacterial culture was added and incubated at $30^{\circ} \mathrm{C}$ and 110 r.p.m. After 4-7 days, sporulation of plates was examined by eye. Light microscopy and test for survival of bacteria was carried out after staining with LIVE/DEAD BacLight fluorescent dyes (Invitrogen Corporation, Karlsruhe, Germany).

Shotgun sequencing of the $\mathrm{B}$. rhizoxinia genome Shotgun sequencing of $B$. rhizoxinica genomic DNA was carried out by GATC Biotech (Konstanz, Germany). A shotgun library of an average insert size of $3 \mathrm{~kb}$ was constructed, as well as a cosmid library with an average insert size of $36 \mathrm{~kb}$. In total, 36000 shotgun clones and 2000 cosmid clones were end sequenced by Sanger technology and the 
obtained reads were assembled using ARACHNE 3 whole genome assembler (Jaffe et al., 2003).

\section{Phylogenetic analysis}

For phylogenetic analysis, protein sequences were aligned by the ClustalW algorithm implemented in the MEGA 3.1 software package (Kumar et al., 2004). The obtained alignment blocks were trimmed and used for tree-construction by the neighbor-joining method. 10000 bootstrap replicates were run to estimate reliability of the inferred groups.

\section{In silico protein sequence analysis}

Prediction of secondary structure elements was conducted using PSIPRED secondary structure prediction server (Bryson et al., 2005), prediction of transmembrane helix structures by TMHMM server version 2.0 (Krogh et al., 2001). Prediction of potentially type III secreted effector proteins was carried out by using T3SS PREDICTION server (Löwer and Schneider, 2009) and the EFFECTIVE T3 prediction tool (Arnold et al., 2009).

\section{Gene expression assay}

RNA was isolated using the RiboPure Bacteria or RiboPure Yeast Kit (Ambion, Austin, TX, USA) following the manufacturers' instructions either from pure bacterial culture or from cocultivation of B. rhizoxinica, and its host after sporulation could be observed with the wild-type bacteria. In total, $200 \mathrm{ng}$ of DNAse-treated RNA served as template for cDNA synthesis for $1 \mathrm{~h}$ at $37^{\circ} \mathrm{C}$ using Omnicscript reverse transcriptase (Qiagen, Hilden, Germany) and random hexamer primers. Real-time PCR was performed on an Eppendorf (Hamburg, Germany) realplex mastercyler in triplicate for each sample, and a control reaction without enzyme was included for each sample. The $16 \mathrm{~S}$ gene was used as an internal standard for calculation of expression levels and normalization. In total, $1 \mu \mathrm{l}$ of cDNA was used for amplification with the DyNAmo Flash Sybr Green qPCR Kit (NEB, Ipswich, UK) with specific primers (Table 2) for sctC (qRTsctC_fw, qRTsctC_rw), sctT (qRTsctT_fw, qRTfsctT_rw), as well as ORF 3 (qRT 3_FW_in, qRT 3_RV_in) and ORF 4 (qRT 4_FW_in, qRT 4_RV_in). For cycling parameters we followed the manufacturers' protocol. Controls without added template were included for each primer pair. Cycle threshold (Ct) values were calculated by the realplex software and used for quantification of expression levels via the $2^{-\Delta \Delta \mathrm{Ct}}$ method (Livak and Schmittgen, 2001).

\section{Acknowledgements}

We thank A Thywissen for assistance with microscopy, K Graupner for PCR screening of mutants, and G-M Schwinger for maintenance of fungal strains. This research was supported by the excellence graduate school Jena School for Microbial Communication (JSMC), the International Leibniz Research School for Microbial and Biomolecular Interactions (ILRS) and the HKI.

\section{References}

Arnold R, Brandmaier S, Kleine F, Tischler P, Heinz E, Behrens S et al. (2009). Sequence-based prediction of type III secreted proteins. PLoS Pathog 5: e1000376.

Barrett AR, Kang Y, Inamasu KS, Son MS, Vukovich JM, Hoang TT. (2008). Genetic tools for allelic replacement in Burkholderia species. Appl Environ Microbiol 74: 4498-4508.

Bright M, Bulgheresi S. (2010). A complex journey: transmission of microbial symbionts. Nat Rev Microbiol 8: 218-230.

Bryson K, McGuffin LJ, Marsden RL, Ward JJ, Sodhi JS, Jones DT. (2005). Protein structure prediction servers at University College London. Nucl Acids Res 33: W36-W38.

Büttner D, He SY. (2009). Type III protein secretion in plant pathogenic bacteria. Plant Physiol 150: 1656-1664.

Coenye T, Vandamme P. (2003). Diversity and significance of Burkholderia species occupying diverse ecological niches. Environ Microbiol 5: 719-729.

Cornelis GR. (2002). The Yersinia Ysc-Yop 'type III' weaponry. Nat Rev Mol Cell Biol 3: 742-752.

Cornelis GR. (2006). The type III secretion injectisome. Nat Rev Microbiol 4: 811-825.

Dale C, Moran NA. (2006). Molecular interactions between bacterial symbionts and their hosts. Cell 126: 453-465.

Dale C, Plague GR, Wang B, Ochman H, Moran NA. (2002). Type III secretion systems and the evolution of mutualistic endosymbiosis. Proc Natl Acad Sci USA 99: $12397-12402$.

Dale C, Young SA, Haydon DT, Welburn SC. (2001). The insect endosymbiont Sodalis glossinidius utilizes a type III secretion system for cell invasion. Proc Natl Acad Sci USA 98: 1883-1888.

Deakin WJ, Broughton WJ. (2009). Symbiotic use of pathogenic strategies: rhizobial protein secretion systems. Nat Rev Microbiol 7: 312-320.

Gamper M, Kast P. (2005). Strategy for chromosomal gene targeting in RecA-deficient Escherichia coli strains. BioTechniques 38: 405-408.

Genin S, Boucher C. (2004). Lessons learned from the genome analysis of Ralstonia solanacearum. Annu Rev Phytopathol 42: 107.

Ghosh P. (2004). Process of protein transport by the type III secretion system. Microbiol Mol Biol Rev 68: 771-795.

Grant SR, Fisher EJ, Chang JH, Mole BM, Dangl JL. (2006). Subterfuge and manipulation: type III effector proteins of phytopathogenic bacteria. Annu rev microbiol 60: $425-449$.

Hueck CJ. (1998). Type III protein secretion systems in bacterial pathogens of animals and plants. Microbiol Mol Biol Rev 62: 379-433.

Jaffe DB, Butler J, Gnerre S, Mauceli E, Lindblad-Toh K, Mesirov JP et al. (2003). Whole-genome sequence assembly for mammalian genomes: Arachne 2 . Genome Res 13: 91-96.

Kim HS, Schell MA, Yu Y, Ulrich RL, Sarria SH, Nierman WC et al. (2005). Bacterial genome adaptation to 
niches: divergence of the potential virulence genes in three Burkholderia species of different survival strategies. BMC Genomics 6: 174.

Kobayashi DY, Crouch JA. (2009). Bacterial/fungal interactions: from pathogens to mutualistic endosymbionts. Annu Rev Phytopathol 47: 63-82.

Krogh A, Larsson B, Von Heijne G, Sonnhammer ELL. (2001). Predicting transmembrane protein topology with a hidden Markov model: application to complete genomes. J Mol Biol 305: 567-580.

Kubori T, Matsushima Y, Nakamura D, Uralil J, Lara-Tejero M, Sukhan A et al. (1998). Supramolecular structure of the Salmonella typhimurium type III protein secretion system. Science 280: 602-605.

Kumar S, Tamura K, Nei M. (2004). MEGA3: Integrated software for molecular evolutionary genetics analysis and sequence alignment. Brief Bioinform 5: 150-163.

Lackner G, Moebius N, Scherlach K, Partida-Martinez LP, Winkler R, Schmitt I et al. (2009a). Global distribution and evolution of a toxinogenic Burkholderia-Rhizopus symbiosis. Appl Environ Microbiol 75: 2982-2986.

Lackner G, Partida-Martinez LP, Hertweck C. (2009b). Endofungal bacteria as producers of mycotoxins. Trends Microbiol 17: 570-576.

Livak KJ, Schmittgen TD. (2001). Analysis of relative gene expression data using real-time quantitative PCR and the $2^{-\Delta \Delta \mathrm{CT}}$ method. Methods 25: 402-408.

Löwer M, Schneider G. (2009). Prediction of type III secretion signals in genomes of gram-negative bacteria. PLOS ONE 4: e5917.

Moran NA. (2006). Symbiosis. Curr Biol 16: R866-R871.

Mukaihara T, Tamura N, Murata Y, Iwabuchi M. (2004). Genetic screening of Hrp type III-related pathogenicity genes controlled by the HrpB transcriptional activator in Ralstonia solanacearum. Mol Microbiol 54: 863-875.

Partida-Martinez LP, Hertweck C. (2005). Pathogenic fungus harbours endosymbiotic bacteria for toxin production. Nature 437: 884-888.

Partida-Martinez LP, Hertweck C. (2007). A gene cluster encoding rhizoxin biosynthesis in 'Burkholderia rhizoxina', the bacterial endosymbiont of the fungus Rhizopus microsporus. Chembiochem 8: 41-45.

Partida-Martinez LP, Groth I, Schmitt I, Richter W, Roth M, Hertweck C. (2007a). Burkholderia rhizoxinica sp. nov. and Burkholderia endofungorum sp. nov., bacterial endosymbionts of the plant-pathogenic fungus Rhizopus microsporus. Int J Syst Evol Microbiol 57: 2583-2590.

Partida-Martinez LP, Monajembashi S, Greulich KO, Hertweck C. (2007b). Endosymbiont-dependent host reproduction maintains bacterial-fungal mutualism. Curr Biol 17: 773-777.
Poueymiro M, Genin S. (2009). Secreted proteins from Ralstonia solanacearum: a hundred tricks to kill a plant. Curr Opin Microbiol 12: 44-52.

Preston GM. (2007). Metropolitan microbes: type III secretion in multihost symbionts. Cell Host Microbe 2: 291-294.

Rainbow L, Hart CA, Winstanley C. (2002). Distribution of type III secretion gene clusters in Burkholderia pseudomallei, B. thailandensis and B. mallei. J Med Microbiol 51: 374-384.

Rezzonico F, Binder C, Défago G, Moënne-Loccoz Y. (2005). The type III secretion system of biocontrol Pseudomonas fluorescens KD targets the phytopathogenic Chromista Pythium ultimum and promotes cucumber protection. Mol Plant Microbe Interact 18: 991-1001.

Scherlach K, Partida-Martinez LP, Dahse H-M, Hertweck C. (2006). Antimitotic rhizoxin derivatives from a cultured bacterial endosymbiont of the rice pathogenic fungus Rhizopus microsporus. J Am Chem Soc 128: 11529-11536.

Schmitt I, Partida-Martinez LP, Winkler R, Voigt K, Einax E, Dölz F et al. (2008). Evolution of host resistance in a toxin-producing bacterial-fungal alliance. ISME J 2: 632-641.

Tarkka MT, Sarniguet A, Frey-Klett P. (2009). Interkingdom encounters: recent advances in molecular bacterium-fungus interactions. Curr Genet 55: 233-243.

Van Gijsegem F, Gough C, Zischek C, Niqueux E, Arlat M, Genin S et al. (1995). The hrp gene locus of Pseudomonas solanacearum, which controls the production of a type III secretion system, encodes eight proteins related to components of the bacterial flagellar biogenesis complex. Mol Microbiol 15: 1095-1114.

Van Gijsegem F, Vasse J, De Rycke R, Castello P, Boucher C. (2002). Genetic dissection of the Ralstonia solanacearum hrp gene cluster reveals that the HrpV and HrpX proteins are required for Hrp pilus assembly. Mol Microbiol 44: 935.

Viallard V, Poirier I, Cournoyer B, Haurat J, Wiebkin S, Ophel-Keller K et al. (1998). Burkholderia graminis sp. nov., a rhizospheric Burkholderia species, and reassessment of (Pseudomonas) phenazinium, (Pseudomonas) pyrrocinia, (Pseudomonas) glathei as Burkholderia. Int J Syst Bacteriol 48: 549-563.

Warawa J, Woods DE. (2005). Type III secretion system cluster 3 is required for maximal virulence of Burkholderia pseudomallei in a hamster infection model. FEMS Microbiol Lett 242: 101-108.

Zhou JM, Chai J. (2008). Plant pathogenic bacterial type III effectors subdue host responses. Curr Opin Microbiol 11: 179-185.

Supplementary Information accompanies the paper on The ISME Journal website (http://www.nature.com/ismej) 